\section{Heroingestützte Therapie für Opiatabhängige - weder Allheilmittel noch Teufelswerk}

Zusammenfassung: Ethische, wissenschaftliche und praktische Probleme bei der Behandlung von Opiatabhängigen unter Einsatz von Heroin als Substitutionsmittel werden erörtert. Diese Probleme ergeben sich zum einen aus der Tatsache, dass Heroinversuche derzeit nicht als klassische biochemische Doppelblindversuche durchgeführt und dass Konfundierungen mit anderen Variablen nicht ausgeschlossen werden können (z.B. Dauer der Betreuung, Reaktivität). Zum anderen sind Heroinversuche in den meisten Ländern stark ideologisiert und deshalb muss mit verschiedenen praktischen Problemen bei der Umsetzung gerechnet werden. Beide Punkte werden an aktuellen Beispielen aus verschiedenen Ländern illustriert. In den Schlussfolgerungen plädieren die Autoren für einen rationaleren Umgang beim Einsatz von Heroin in der Substitutionsbehandlung von Opiatabhängigen und für den Aufbau von Behandlungssystemen in diesem Bereich, die Therapien mit verschiedenen Methoden (unter Einschluss anderer Substitutionsbehandlungen und Abstinenztherapien) anbieten, wobei aufgrund von wissenschaftlichen Ergebnissen eine individuelle Zuordnung von Patient und Therapie vorgenommen werden soll.

Schlüsselwörter: Heroin - Opiatabhängigkeit - Substitutionsbehandlung - Experiment - ethische Probleme

Heroin Supported Therapy for Opioid Addicts - neither Panacea nor Devilry: Ethical, scientifical and practical problems concerning substitution treatment for opiate addicts involving heroin as substitution agent are discussed. Main scientific problems concern the impossibility to conduct a heroin trial as a classic biochemical double-blind experiment and the impossibility to completely rule out confounding (e.g. duration of contact with therapists, reactivity). In addition, there are political and ideological debates concerning such trials, which cause practical problems. Both points are illustrated with examples from different countries. The conclusions argue for a more rational consideration of the application of heroin within substitution treatment for opiate addicts. Also, we should try to build treatment systems in this field, where different kinds of therapeutic approaches are offered (including the use of other substitution agents and abstinence-oriented therapies) and where matching of patient and therapy will be based on scientific knowledge.

Suchttherapie 2000; 1: 57-62

(c) Georg Thieme Verlag Stuttgart · New York ISSN 1439-9903
Jürgen Rehm¹, 2, 3,4 , Benedikt Fischer2, 3, 5

1 Institut für Suchtforschung, Zürich, Schweiz

2 Department of Public Health Sciences, University of Toronto, Toronto, Kanada

3 Social, Prevention and Health Policy Research Department, Centre for Addiction and Mental Health, Toronto, Kanada

${ }^{4}$ World Health Organization (WHO), Genf, Schweiz

${ }^{5}$ Centre of Criminology, University of Toronto

Key words: Heroin - Opiate Addiction - Substitution Treatment - Controlled Clinical Trial - Ethical Problems

\section{Die Geschichte der Opiatverschreibung}

Die Geschichte der klinischen Opiatverschreibung in der Behandlung von Opiatabhängigen ist nahezu ein Jahrhundert alt. Die Morphin-Kliniken in Shreveport, USA, verabreichten zwischen 1919 und 1923 legale Opiate an Morphiumabhängige und das Konzept einer therapeutischen Betäubungsmittelverabreichung wurde nach der Ausbreitung des Heroinabhängigkeitsproblems in den 40er- und 50er Jahren in Nordamerika intensiv in medizinischen und politischen Kreisen diskutiert, aber nie systematisch durchgesetzt $[9,15,21,26]$. Medizin, Strafverfolgung und Politik insistierten auf der Loslösung von Betäubungsmittelabhängigen von ihrer Sucht (Abstinenzparadigma) und lehnten in der Mehrheit Substitutionsansätze grundsätzlich ab.

Im Zuge der auch immer deutlicher von der Fachöffentlichkeit als nicht zufriedenstellend perzipierten unzulänglichen Ergebnisse traditioneller, abstinenzorientierter Behandlungsmethoden im Lichte einer sich ausbreitenden Heroinabhängigkeitsproblematik setzten die pharmakotherapeutischen Versuche von Dole und Nyswander [10] in USA und - weniger bekannt - Halliday [23] in Kanada mit dem synthetischen Oral-Opiat Methadon zu Anfang der 60er Jahre ein bahnbrechendes Signal. Sie zeigten, dass die Methadonsubstitutionstherapie durch Blockieren der entsprechenden Rezeptoren den Drang der meisten Opiatabhängigen zu illegalem Heroinkonsum stark minderte und dadurch negative Konsequenzen wie Kriminalität, Gesundheitsprobleme und Tod deutlich zu senken versprach. Die Methadonverabreichung freilich bot weitere, höchst willkommene Charakteristika für eine Suchttherapie: Das Substitut konnte oral verabreicht werden und vermittelte dem Patienten keinen nachhaltigen psychotropen Effekt und war damit politisch und fachlich um ein Vielfaches akzeptabler als z.B. injiziertes Morphium oder Heroin.

In Nordamerika wurde die Verbreitung der Methadonsubstitution als grundständige medizinische Behandlung nach anfänglicher Euphorie zu Anfang der 70er Jahre abrupt gestoppt, durch umfassende Behandlungsregulatorien drastisch eingeschränkt und ihre Verfechter in der therapeutischen und wissenschaftlichen Szene sozial isoliert $[9,15]$. Drogenkontrollbehörden, aber auch gewichtige Interessensfraktionen 
der Suchtmedizin lamentierten über Diversions-, Gesundheits- und ideologische Risiken sowie Schwächen dieser „Suchterhaltungstherapie“ und übten Druck auf Politik und Praxis aus, sich (wieder) auf die Prinzipien wirklicher (das heißt drogenfreier) Therapie zu verlassen. Obwohl in Details der Chronologie und hinsichtlich der Teilnehmer am Suchtdiskurs nicht identisch, verlief die bis vor kurzem äußerst verhaltene und limitierte Entwicklung der OpiatverschreibungsTherapie in vielen europäischen Ländern (wie z.B. Deutschland, Österreich, Schweden, Schweiz) durchaus ähnlich (für Überblicke siehe $[12,28,43])$. Eine nennenswerte Ausnahme bildet in Europa lediglich Großbritannien, wo Opiatverschreibung, auch in Form von Heroin, seit jeher in der allgemeinen Primärkrankenversorgung verankert gewesen ist, aber doch im Laufe der Jahre beträchtliche Einschränkungen erfahren musste [31].

Erst die Eskalation des „Injection Drug Use“(IDU)-Problems und seiner explodierenden Schadensfolgen und sozialen Kosten, vor allem durch die HIV- und Hepatitis-Epidemien, massive Todesraten durch Überdosierung in urbanen Zentren $[16,34,42]$, aber auch eine gewisse Ent-Ideologisierung der Betrachtung und des Denkens über Drogensucht haben eine Ausbreitung der Methadon-Behandlung (Kanada, Schweiz) bzw. ihre legale Initiierung in Ländern ermöglicht, wo sie lange verboten war (wie z.B. Deutschland oder Österreich).

Offenkundige Grenzen der Wirksamkeit der Methadonbehandlung - zum einen in der Anziehung neuer Patienten, zum anderen in der Haltung und wirksamen Therapie von Patienten - sind seit langem bekannt und empirisch gut belegt $[17,19]$. Daran werden nach heutiger Erkenntnis auch die in der klinischen Erprobungsphase stehenden „neuen“ Substitutionsmittel wie Buprenorphin- oder LAAM-Varianten nichts drastisch ändern oder verbessern. Es überrascht daher nicht, dass Forderung und Diskussion der Erprobung einer Substitutionstherapie mit Hilfe der ursprünglichen Wahldrogen als einzig wirklichem „Ersatz“ für Non-Responder schon zu den Ursprüngen der Methadonbehandlung zurückreichen, wie beispielsweise Anstrengungen für Heroinverschreibungsversuche als „Last Resort Treatment“ aus den USA und Kanada von vor fast 30 Jahren illustrieren $[19,29]$. Auch in Holland und Großbritannien experimentierte man vereinzelt, aber recht unsystematisch während dieser Zeit mit der Heroinvergabe [28].

Die massiven negativen Konsequenzen von Heroinabhängigkeit in fortgeschrittenen Marktwirtschaften sowie die eng begrenzte Wirksamkeit traditioneller Therapien hat schließlich im Laufe der 90er Jahre zur ersten - kontrovers diskutierten Generation wissenschaftlicher Vorstöße beziehungsweise Projekte in der experimentellen Heroinverschreibung in der Schweiz, Australien und den Niederlanden $[1,6,28]$ geführt.

\section{Ethische Probleme bei Heroinverschreibung}

Die ethischen Probleme von Heroinverschreibungen sind ähnlich wie diejenigen anderer Substitutionstherapien: Sie können unterteilt werden in Probleme auf individueller und sozialer Ebene. Individualethisch stellt sich die Kernfrage, ob Süchtige genug Automie und „freien Willen“ haben, um für sich die beste verfügbare Therapie auswählen zu können, oder ob nicht gerade wegen der suchtspezifischen Defizite eine Therapie, z.B. eine Abstinenztherapie, von dazu autorisierten Instanzen verfügt werden sollte. Die derzeitige Auffassung in der medizinischen und juristischen Fachliteratur [2] hierzu favorisiert die autonome Willensfähigkeit Süchtiger, gerade auch im Vergleich mit anderen psychiatrisch definierten Krankheiten (vgl. dazu [11]). In diesem Zusammenhang unbedingt beachtenswert ist der Umstand, dass zwangsverfügte Abstinenztherapien im Allgemeinen keine besseren $\mathrm{Er}$ gebnisse zeitigen als Substitutionstherapien.

Die Sozialethik stellt zur Frage der Substitutionstherapien zwei Probleme in den Vordergrund. Hat der Staat ein Recht, soziale Gesichtspunkte (d.h. Gesichtspunkte, die die Gesundheit des Individuums nicht unmittelbar berühren) wie die Verminderung von Kriminalität, HIV-Verbreitung oder soziale Kosten als wichtigste Ergebnisse einer medizinischen Therapie zu betrachten? Genau dies wird durch die Festlegung der Hauptzielkriterien von Heroin- oder Methadonstudien ja so vorausgesetzt, z.B. auch im bekanntesten Projekt der Schweiz [44]. Wir glauben, dass es dafür Argumente gibt: Zum einen sind Ressourcen nicht nur in modernen Gesundheitssystemen knapp, d.h., staatliche Stellen müssen an allen Therapien interessiert sein, die die gesellschaftlichen Gesamtkosten senken. Zum zweiten geht es Opiatabhängigen im Allgemeinen nach Eintritt in die Therapie gesundheitlich besser als vorher ( = Nachweis der Effektivität) und auch die subjektiv empfundene Lebensqualität steigt. Und zum dritten kann argumentiert werden, dass der schlechte gesundheitliche Zustand von unbehandelten Opiatabhängigen [18] zu einem durchaus nicht geringen Teil die Folge staatlich definierter Randbedingungen für das Suchtverhalten darstellt. Insbesondere die Illegalität der Opiatabhängigkeit und die damit verbundenen Lebensgewohnheiten $[13,30,36]$ sind hier zu nennen.

Ein verwandtes ethisches Problem betrifft die Zuteilung von knappen Ressourcen in aufwendige und teure Heroinverschreibungs-Versuche. Wären solche Ressourcen nicht besser in anderen Bereichen des Gesundheitswesens angelegt? Derzeit fehlt aber ein klarer Maßstab, der die Kosteneffektivität verschiedener gesundheitspolitischer Maßnahmen zweifelsfrei vergleichen ließe. Die Frage kann deshalb zum heutigen Zeitpunkt nicht eindeutig beantwortet werden. Es ist zu hoffen, dass die Weiterentwicklung von Maßen wie DALY (disability adjusted life years [32]) zu einem akzeptierten Standard führt und dass in Zukunft generell rationalere, d.h. auf empirischen Grundlagen basierende und mit klaren Maßstäben und nach informierter Diskussion gefällte gesundheitspolitische Entscheidungen gefällt werden. Gegenwärtig dominieren leider noch ideologiegespeiste Debatten und dies gilt besonders im gesundheitspolitischen Diskurs über illegale Drogen (siehe unten).

\section{Wissenschaftliche Probleme bei Heroinschreibung}

In einer solchen Gemengelage werden dann - wie es den Anschein erweckt - Versuche zur Heroinverschreibung auch schon mal absichtlich „naiv“ geplant, so als ob Heroinsubstitutionsversuche $\mathrm{x}$-beliebige Experimente von pharmakologisch wirksamen Substanzen wären, die unter isolierten Laborkonditionen zu erforschen seien. Davon kann natürlich keine Rede sein. Doppelblindversuche mit Plazebokontrollen sind ebenso wenig möglich oder sinnvoll wie Doppelblindvergleiche zu langfristig wirkenden Agonisten wie z.B. Metha- 
don. Selbst Doppelblindvergleiche gegen kurzfristig wirkende Substanzen wie Hydromorphon (Dilaudid) scheinen nach neuesten Erkenntnissen, wie erste Ergebnisse einer Pilotstudie in Toronto und Baltimore zeigen, ausgeschlossen (persönliche Mitteilung von Dr. David Marsh), da die Klienten in der Lage sind, Heroin zu erkennen. Studiendesigns nach Maßgabe von zulassungsrelevanten Pharmastudien sind demnach strenggenommen nicht möglich.

Hinzu kommt, dass aufgrund der kurzfristigen Wirkung des Heroins Klienten meist dreimal pro Tag an der Vergabestelle erscheinen müssen [44]. Hier zeigen sich große Unterschiede zur Methadonbehandlung alleine schon im zeitlichen Ausmaß des Kontaktes zum sozialtherapeutischen Personal der Verschreibungsstelle, der über längere Zeit schwerlich balanciert bzw. wissenschaftlich kontrolliert werden kann. Die Vergleichbarkeit der Gruppen ist nicht mehr gegeben und damit kann die pharmakologische Wirkung nicht mehr von der potenziellen Wirkung dieser häufigen Kontakte getrennt werden $[1,39]$.

Schließlich ergibt sich das Problem, dass Heroin nach den meisten Studienprotokollen an Personen untersucht wird, die mit Methadonbehandlung nicht zurechtkamen (Therapieabbrecher), und dass dann aber in den meisten Fällen mit eben dieser Substanz verglichen werden soll. Dies führt dazu, dass in Ländern mit gutem Angebot an qualitativ hochwertigen Methadonsubstitutionstherapien die Klienten sich zwar melden, aber nach Randomisierung in einen Methadonarm den Versuch wieder verlassen (z.B. Schweiz). In Ländern ohne ausreichendes Angebot von Methadon oder mit Therapieregimen, die von der Dosierung her deutlich unter dem Optimum liegen, ist dieses Phänomen weniger häufig zu erwarten (zum Beispiel für den Fall, dass in Nordamerika das NAOMI-Protokoll implementiert würde; s.u.).

Zwar nicht spezifisch für Heroinversuche, aber virulent wie für alle Projekte, die in der Öffentlichkeit umstritten sind und deshalb intensiv diskutiert werden, sind Probleme der Reaktivität $[3,39]$. Nach allen psychologischen Erkenntnissen wäre es eine Fiktion zu glauben, dass die Teilnehmer in einem kontrollierten Verschreibungsexperiment von solchen Diskussionen unbeeinflusst blieben. In unseren Gesellschaften mit hoher Medienwirksamkeit hat sich gezeigt, dass nach Serien über den Suizid eines Schülers die Suizidquote ebenso stieg (z.B. [24]) wie die Anzahl von Klagen von potenziellen Holzschutzmittelgeschädigten nach entsprechenden anderen Berichten. Man kann also davon ausgehen, dass die Ergebnisse eines „heiß“ diskutierten Heroinverschreibungsversuchs durchaus durch die öffentliche Diskussion beeinflusst werden.

\section{Die politische Dimension}

Es steht außer Frage, dass es bei Heroinversuchen nicht ausschließlich um suchtklinische Praktiken oder Wissenschaft geht, sondern in hohem Maße (wenn nicht vorrangig!) um Politik, Interessen und damit auch um Ideologie und Moral. Die meisten Gesellschaften tun sich nach wie vor mit dem Konzept langfristiger Substitutionstherapie im Allgemeinen schwer, da ihr Etiketten anhaften wie „Kapitulation vor der Sucht“ oder „Legalisierung“ sowie der Vorwurf, aus dem Staat einen Drogendealer zu machen, der den Patienten im Falle der
Heroinverschreibung jetzt auch noch mit gefährlichen und euphorisierenden Drogen versorgt [35, 37, 41].

Kein Land kann und wird aber mit Heroinverschreibung experimentieren oder diese gar als Regeltherapie einsetzen, ohne dass dieser Schritt explizit von Staat und Regierung getragen oder unterstützt wird (siehe die verschiedentlich orientierten Fallbeispiele der Schweiz und Australiens [4, 38]).

Das entscheidende Instrument für diese machtpolitischen Entscheidungen sind im Zweifelsfall, wie so oft, die finanziellen Ressourcen. In europäischen Gesellschaften kann im Allgemeinen nur der Staat selbst die äußerst teuren Heroinversuche finanzieren, in Nordamerika kontrollieren die Regierungen die Budgets der einschlägigen Forschungs(förderungs)institute, ohne die keine respektable wissenschaftliche Forschung in diesem Bereich unternommen werden kann.

Auf der anderen Seite ist Heroinverschreibung mit Sicherheit kein Thema, mit dem sich Wahlen gewinnen lassen. Im Gegenteil birgt sie ein deutliches Risiko, zu lästigen Kontroversen zu führen, mit denen kaum einer relevanten Lobby gedient ist. Auf lokaler politischer und praktisch-therapeutischer Ebene wiederum ist man natürlich besorgt, dass die befürchtete vehemente Anziehungskraft von legaler Heroinabgabe mögliche Versuchszentren in unkontrollierbare Magnete für noch mehr illegale Konsumenten und weiter wuchernde Drogenszenen verwandelt (das „Honey Pot“-Phänomen, vgl. [4,38]). Und vor einem bürgerlich-zivilen Widerstand nach dem St.-FloriansPrinzip („prinzipiell ja, aber nur nicht vor meiner Haustür“) ist natürlich auch die Heroinverschreibung nicht gefeit.

Aber selbst Heroinverschreibungswillige Staaten sind in ihrer Souveränität, solche Versuche durchzuführen, erheblich beschnitten beziehungsweise unterliegen einem nicht unerheblichen Einschüchterungsfaktor von außen. Der Apparat der internationalen Betäubungsmittelkontrolle, wie er sich im letzten Jahrhundert herausgebildet hat, ist traditionell von drogenfreier Repressionsideologie sowie Strafverfolgungsinteresse amerikanischen Ursprungs geprägt und hat mit den internationalen Konventionen ein anachronistisches, gleichwohl juristisch wirksames Kontrollnetz gesponnen $[40,45]$. Dieses kann zwar mühsam über die Wissenschafts-Paragraphen und durch Diplomatie auf hoher politischer Ebene umschifft werden, führt aber zu einer weiteren Ebene aufreibender Politisierung. Dass auf dieser Basis dann Institutionen wie das International Narcotics Control Board (INCB) über die Angemessenheit und Aussagekraft von klinischen Heroinverschreibungs-Versuchen befinden (vgl. [25], Para. 367-369), mag zwar auf den ersten Eindruck paradox und irrelevant zugleich erscheinen, hat aber ganz reale Konsequenzen. Die australischen Heroin-Versuchsvorbereitungen beispielsweise, seit zehn Jahren mit großem Aufwand betrieben, sind bislang wiederholt kurz vor Studienbeginn doch noch von höchster politischer Regierungsebene gestoppt worden, wobei die Einflüsse der internationalen Drogenkontrollbehörden sowie der USA hierfür im Hintergrund als maßgeblich anzuerkennen sind $[4,33]$.

Aber es spielen auch subtilere Faktoren eine Rolle. In einigen Ländern haben sich z.B. die Praktizierung traditioneller OpiatPharmakotherapie (zumeist Methadon) und die dazugehörigen psychosozialen Dienstleistungen zu einer recht profitab- 
len Spezialindustrie im Bereich allgemeiner Gesundheitsversorgung entwickelt, die sich nun plötzlich unangenehm einer neuen Konkurrenz, aber auch neuen Qualitätsmaßstäben und -erwartungen an die Opiat-Pharmakotherapie ausgesetzt sieht $[7,20,22]$. Ein nicht geringer Teil des Widerstandes gegen klinische Heroinverschreibungsversuche in diesen Ländern muss aus dieser korporatistischen und interessenspolitischen Verfasstheit der Suchtmedizin verstanden werden.

\section{Internationale Versuche: eine kurze Übersicht}

Die nachfolgende Übersicht über die aktuellen Erfahrungen mit Heroinversuchen in ausgewählten Ländern illustriert die obigen Gedanken noch einmal ganz praktisch:

In der Schweiz ist Heroinsubstitutionsbehandlung mittlerweile ein etablierter Bestandteil des Therapiespektrums in der Primärversorgung, wenn auch nur ein quantitativ kleiner Teil verglichen mit der Methadontherapie. Das Therapiespektrum wird zusätzlich durch abstinenzorientierte Behandlungen ergänzt. Die Politisierung der Diskussion um die Opiatbehandlung hat deutlich abgenommen und die Fragestellungen gehen inzwischen eher in Richtung auf differenzialdiagnostische Probleme: Welche Therapie nützt welchen Abhängigen am meisten? Zu diesem Thema wird gegenwärtig für das Jahr 2001 eine große Konsensus-Konferenz vorbereitet.

Die Niederlande begannen im Jahre 1999 mit einem mehrarmigen, kontrollierten Heroinverschreibungs-Versuch, der auf einem randomisierten Cross-over-Design basiert. Die Teilnehmer sind in drei Gruppen unterteilt, von denen jede während mindestens einer von drei 6-Monats-Phasen Heroin (in Kombination mit Oral-Methadon) erhält. In der Kontrollphase wird jeweils die reguläre Methadonbehandlung verabreicht. Der Gesamtversuch ist in zwei separate Teilstudien nach obiger Anordnung unterteilt, von denen eine mit inhalierbarem Heroin, die andere mit intravenösem Heroin experimentiert. Der Versuch findet in einem halben Dutzend Städten mit einer Gesamtzahl von ca. 700 Probanden statt, die aus NonRespondern einer vorangegangenen Methadonbehandlung rekrutiert werden. Zur Evaluation wird ebenfalls über einen so genannten Harm-Index das Hauptzielkriterium nach $\mathrm{Ge}-$ sundheits-, Sozial- und Drogenkonsum-Indikatoren operationalisiert. Einer der wichtigsten Kritikpunkte am niederländischen Versuch ist die Möglichkeit, dass sowohl Verbleiben wie Suchtverhalten der Probanden in den (Methadon)-Kontrollgruppen von deren Kenntnis um die erlebte bzw. noch zu erwartende Heroinversorgung beeinflusst und verfälscht werden [8].

In Deutschland läuft derzeit eine öffentliche Ausschreibung zur wissenschaftlichen Erprobung der ärztlichen Heroinverschreibung, die nach Kritik von externen Begutachtern mittlerweile in der zweiten Runde einer möglichen Vergabe steht. Mit dem Beginn der Versuche wird noch im Jahr 2000 oder zu Beginn des Jahres 2001 gerechnet. Wichtigste Probleme sind für die Designentwicklung gegenwärtig noch die teilweise divergierenden Wunschzettel von Bundesregierung, Stadtverwaltungen bei den teilnehmenden Studienzentren und Forscherteams.
In anderen europäischen Ländern sind Heroinversuche derzeit in der Antragsphase (z.B. Belgien), in Pilotphasen (z.B. Spanien, Großbritannien) oder werden derzeit nicht erwogen (z.B. Schweden, Finnland). Eine allgemeine Übersicht zu Drogenbehandlungssystemen nicht nur in Europa findet sich in Klingemann und Hunt [27].

In Australien hat sich die Wissenschaft schon seit fast zehn Jahren systematisch auf einen Heroinversuch vorbereitet und eingestellt, ist aber schließlich bis heute immer wieder am politischen Veto der Bundesregierung gescheitert, obwohl sich eine Mehrheit der regionalen Gesundheits- und Polizeiminister dafür ausgesprochen hatte. Das eigentliche Versuchsdesign basiert auf einem klassischen randomisierten zweiarmigen Therapievergleich, in dem Probanden entweder der ausschließlichen Methadonbehandlung oder dem aktiven Behandlungsarm zugewiesen werden, in dem injizierbares Heroin und orales Methadon nach Wunsch kombiniert werden können. Nach einer Pilotphase mit einer kleineren Stichprobe sollte der Vollversuch in drei Städten mit insgesamt 500 Patienten zunächst über die Dauer eines Jahres stattfinden (gefolgt von einer Wahlbehandlungsphase für alle Patienten) und über einen Indikatoren-Index zu Gesundheits-, Kriminalitäts- und Drogengebrauchs-Status evaluiert werden. Besonderes Kennzeichen des australischen Versuchsplans ist, dass ein sehr breites Zielgruppenspektrum als Teilnehmerfeld anvisiert wurde (Behandlungs-Naive, Behandlungs-Ausscheider sowie sich in anderer Behandlung befindliche Opiatabhängige; vgl. [5]).

In Nordamerika ist seit zwei Jahren eine Gruppe von Suchttherapeuten und -forschern damit befasst, ein Protokoll für einen multizentrischen Heroinversuch (NAOMI: North American Opiate Medications Initiative) zu entwerfen. Das vorläufige Design sieht einen zweiarmigen, randomisierten Versuch in sechs städtischen Versuchszentren mit jeweils ca. 100 Probanden vor, die aus Non-Respondern vorangegangener (Methadon)-Therapien rekrutiert werden sollen und über die Dauer von zunächst einem Jahr beforscht werden. Die Experimentalgruppe soll eine individualisierte Heroin-MethadonKombination erhalten; die Kontrollgruppe dagegen nur Methadon, wobei unterstrichen wird, dass in beiden Schienen die psychosozialen Begleitbehandlungen in Intensität und Qualität möglichst gleichgehalten werden, um eventuell verfälschende Behandlungseffekte von dieser Ebene her auszuschließen. Das NAOMI-Protokoll verwendet als Zielkriterium für die Studie den Behandlungsverbleib der Patienten über die Versuchsdauer sowie einen kombinierten „Harm-Index“ zur Effektivitätsmessung, wobei auf eine „Intent-to-Treat“Analyse gebaut wird, d.h., es werden alle ursprünglich in den Versuch aufgenommenen Probanden in die Outcome-Evaluation einbezogen. Das Versuchsprojekt ist noch in einem theoretischen Stadium. Als wahrscheinlichste kanadische Versuchszentren gelten Toronto, Montreal und Vancouver, wo das Protokoll mittlerweile auch zur ethischen Begutachtung eingereicht wurde; in den USA sind verschiedene wissenschaftlich-klinische Institutionen und Universitäten an der Versuchsentwicklung beteiligt [14]. Neben der drastischen ideologisch-politischen Hürde, die es vor allem in USA für einen Heroin-Verschreibungsversuch zu überwinden gilt, bereitet in Nordamerika vor allem die Tatsache Probleme, dass das konventionelle Methadon-Behandlungssystem sowohl in Angebotsdichte als auch -struktur eher limitiert und unter- 
entwickelt ist [29]. Nur ca. 25\% aller geschätzten Opiatabhängigen in beiden Ländern befinden sich in Methadon-Behandlung (d.h., diese Rate ist nur halb so hoch wie beispielsweise in der Schweiz [19]), so dass Kritiker der Initiative eines Heroinversuchs auf die Notwendigkeit pochen, zunächst die konventionelle Opiat-Pharmakotherapie auszubauen bzw. zu reformieren. Außerdem sind in den nordamerikanischen Opiatkonsumpopulationen sehr viele Personen von einer zusätzlichen Kokain-Abhängigkeit sowie psychiatrischer Komorbidität betroffen, was die Angemessenheit von Heroinverschreibung als Primärbehandlung für viele Abhängige deutlich infrage stellt.

\section{Schlussbemerkungen}

Was können wir aus dem Gesagten lernen? Zum ersten ist es offensichtlich in keinem der beschriebenen Länder möglich, einen reinen biochemischen Versuch zur Wirksamkeit von Heroin vs. Methadon bzw. einer anderen Substanz durchzuführen. Zum zweiten entstehen die Probleme aus der deutlichen Politisierung und Ideologisierung von Substitutionsbehandlungen mit Heroin, zum dritten aber auch dadurch, dass Heroinversuche relativ teuer sind.

Dennoch ist nach den bisherigen Erkenntnissen längerfristige Substitution mit Heroin nicht nur möglich, sondern auch erfolgreich bei der Verringerung von suchtbedingten negativen Konsequenzen [44]. Deshalb plädieren wir für einen gelasseneren und rationaleren Umgang mit Heroinverschreibungsversuchen. Sie sollten gesehen werden als einer von mehreren möglichen Wegen, um mit durch Drogensucht entstandenen Problemen in etablierten Marktwirtschaften umzugehen. Dabei sollte durch weitere wissenschaftliche Arbeiten am dringlichsten geklärt werden, welche Abhängigen am meisten von Heroin profitieren und welches Gesamtspektrum an Therapieangeboten insgesamt am erfolgreichsten ist. Ein ständiges Replizieren der Erkenntnis aus anderen Ländern, dass Heroinsubstitutionsbehandlungen möglich sind und dass sie kombiniert mit optimaler sozialer Betreuung Erfolg bei der Reduktion von heroininduzierten Problemen zeigen, scheint hier nicht zielführend. Vielmehr sollte experimentell geklärt werden, welche Kombination von pharmakologischer und psychosozialer Therapie für welche Abhängigen am erfolgreichsten ist und wie verschiedene Arten von Therapien am besten zu einem integrierten Behandlungssystem verbunden werden können. Für den Erkenntnisfortschritt der Wissenschaftler wie für die praktische Verbesserung der Therapiesituation in den beteiligten Ländern wäre es dabei mehr als nur „Von Vorteil“, wenn sich dazu die Forscher aus verschiedenen Ländern auf einen gemeinsamen Fragenkatalog und ein aufeinander abgestimmtes Forschungsprogramm einigen könnten.

\section{Literatur}

${ }^{1}$ Ali R, Auriacombe M, Casas M, Cottler L, Farrell M, Kleiber D, Kreuzer A, Ogborne A, Rehm J, Ward P. Report of the external panel on the evaluation of the Swiss Scientific Studies of Medically Prescribed Narcotics to Drug Addicts. Sucht 1999; 45 (3): 160170

${ }^{2}$ Amelung K. Zur Verantwortlichkeit Drogenabhängiger für Selbstschädigungen durch den Gebrauch von Suchtstoffen. Neue Juristische Wochenschrift 1996; 49: 2393-2398
${ }^{3}$ Aronson E, Ellsworth PC, Carlsmith JM, Gonzales MH. Methods of research in social psychology. New York: McGraw-Hill, 1990

${ }^{4}$ Bammer G. The ACT heroin trial: Intellectual, practical and political challenges: The 1996 Leonard Ball Oration. Drug and Alcohol Review 1997; 16: 287-296

5 Bammer G, Crawford D, Dance P, Ostini R, Stevens A. Controlled heroin availability in Australia? How and to what end? International Journal of the Addictions 1995; 30: 991-1007

${ }^{6}$ Bammer G, Dobler-Mikola A, Fleming P, Strang J, Uchtenhagen A. The heroin prescribing debate: Integrating science and politics. Science 1999; 284: 1277-1278

${ }^{7}$ Bell J, Ward J, Mattick R, Hay A, Chan J, Hall W. An evaluation of private methadone clinics: National Drug Strategy Research Report No.4. Canberra: Australian Government Publishing Service, 1995

${ }^{8} \mathrm{CCBH}$. Investigating the Medical Prescription of Heroin. A Randomized Trial to Evaluate the Effectiveness of Medically CoPrescribed Heroin and Oral Methadone, Compared to Oral Methadone Alone in Chronic, Treatment Refractory Heroin Addicts. Utrecht: CCBH, 1997

${ }^{9}$ Courtwright DT. A Century of American Narcotics Policy. In: Gerstein DR, Harwood HJ (Hrsg). Treating Drug Problems (Institute of Medicine, vol. 2), Washington: National Academy Press, 1992

10 Dole V, Nyswander M. A medical treatment for diacetylmorphine (heroin) addiction. Journal of the American Medical Association 1965; 193: 646-650

${ }^{11}$ Engelhardt HT. Autonomie und Selbstbestimmung: Grundlegende Konzepte der Bioethik in der Psychiatrie. In: Pöldinger W, Wagner W (Hrsg). Ethik in der Psychiatrie, Berlin Heidelberg New York: Spinger, 1991: 61-71

${ }^{12}$ Farrell M. A review of the legislation, regulation and delivery of methadone in 12 Member States of the European Union. London: European Commission, 1995; ISBN 92-8276-094-4

${ }^{13}$ Faupel C. Shooting Dope: Career Patterns of Hard-Core Heroin Users. Gainesville: University of Florida Press, 1991

${ }^{14}$ Fischer B. Heroinabhängigkeit, -behandlung und -politik in Kanada: Geschichte, Gegenwart, und Perspektiven für einen Heroin-Verschreibungsversuch. In: Westermann B, Bellmann G, Jellinek C (Hrsg). Heroinverschreibung - Wirkungen und Nebenwirkungen, Weinheim: Deutscher Studienverlag, 1999

${ }^{15}$ Fischer B. Prescriptions, Power and Politics: The turbulent history of methadone maintenance in Canada. Journal of Public Health Policy, 21 (3): in press

${ }^{16}$ Fischer B, Kendall P, Rehm J, Room R. Charting WHO-goals for licit and illicit drugs for the year 2000: Are we „on track“? Public Health 1997; 111: 271-275

${ }^{17}$ Fischer B, Kirst M, Rehm J, Marsh D, Bondy S, Tyndall M (forthcoming). The phenomenon of so-called 'other drug use' among opiate addicts in the North American context: Evidence, consequences, questions. In: Westermann B, Jellinek C, Bellman GU (Hrsg). Beigebrauch: Offene Grenzen der Substitution, Beltz: Deutscher Studienverlag,

18 Fischer B, Medved W, Gliksman L, Rehm J. Illicit opiate use in Toronto: A profile of current users. Addiction Research 1999; 7 (5): 377-415

${ }^{19}$ Fischer B, Rehm J. The case for a heroin substitution treatment trial in Canada. Canadian Journal of Public Health 1997; 88: 367-370

${ }^{20}$ Fleming P, Morey J, Charlton P. Is the policy of encouraging general practitioners to prescribe opiates flawed? International Journal of Drug Policy 1997; 8 (4): 172-177

21 Giffen J, Endicott S, Lambert S. Panic and Indifference - The Politics of Canada's Drug Laws. Ottawa: Canadian Centre on Substance Abuse, 1991

22 Greenwood J. Persuading general practitioners to prescribe good husbandry or a recipe for chaos? British Journal of Addiction 1992; 87: 567-575 
${ }^{23}$ Halliday R. Management of the Narcotic Addict. British Columbia Medical Journal 1963; 5: 412-414

${ }^{24}$ Hassan R. Effects of newspaper stories on the incidence of suicide in Australia: A research note. Aust N Z J Psychiatry 1995; 29 (3): 480-483

25 International Narcotics Control Board (INCB). Report of the International Narcotics Control Board for 1997. Vienna: INCB, 1998

${ }^{26}$ Jaffe J. The History and Current Status of Opiate Agonist Treatment. Paper presented at the NIH Consensus Development Conference of Effective Medical Treatment of Heroin Addiction, Nov. 17-19, Bethesda, MD, 1997

${ }^{27}$ Klingemann H, Hunt G. Drug Treatment Systems in an International Perspective: Drugs, Demons, and Delinquents. London: Sage, 1998

${ }^{28}$ Krausz M, Uchtenhagen A, van den Brink W. Medically indicated heroin prescription in the treatment of drug addicts: Clinical trials and developments in research in Europe. Sucht 1999; 45 (3): 171-186

${ }^{29}$ Kuo I, Fischer B, Vlahov D. Consideration of a North American Heroin-Assisted Trial for the Treatment of Opiate-Dependent Individuals. International Journal of Drug Policy, (in press)

${ }^{30}$ Lindesmith A. Addiction and Opiates. Chicago: Aldine Publishing Company, 1947

31 Marsden J, Gossop M, Farrell M, Strang J. Opioid substitution: Critical issues and future directions. Journal of Drug Issues 1998; 28: 243-264

32 Murray CJL, Lopez , A . The global burden of disease. Boston: Harvard School of Public Health, 1996

33 Nadelmann E. Commonsense Drug Policy. Foreign Affairs 1998; 77 (1): $111-126$

${ }^{34}$ Nadelmann E, McNeely J, Drucker E. Harm reduction drug control strategies: A global perspective. In: Lowinson J, Ruiz P, Millman R (Hrsg). Substance Abuse: A Comprehensive Textbook, Baltimore: Williams and Wilkins, 1997: 22-39

${ }^{35}$ Neumeyer J, Schaich-Walch G (eds.). Zwischen Legalisierung und Normalisierung. Ausstiegsszenarien aus der repressiven Drogenpolitik. Marburg: 1992

${ }^{36}$ Nurco D, Shaffer J. Types and characteristics of addicts in the community. Drug and Alcohol Dependence 1982; 9: 43-78

37 Ostrowski J. The moral and practical case for drug legalization. Hofstra Law Review 1990; 18 (3): 607-702

38 Rihs-Middel M. Die Heroinvergabe im Spannungsfeld von Forschung und Praxis. In: Westermann B, Bellman G, Jellinek C (Hrsg). Heroinverschreibung: Wirkungen und Nebenwirkungen, Köln: Deutscher Studien Verlag, 1999: 77-113

${ }^{39}$ Rehm J, Strack F. Kontrolltechniken. In: Hermann T, Tack W (Hrsg). Methodologische Grundlagen der Psychologie. Enzyklopädie der Psychologie, Themenbereich B, Serie 1, Band 1, Göttingen: Hogrefe, 1994: 508-555

40 Room R, Paglia A. The international drug control system in the post-Cold War era. Managing markets or fighting a war? Drug and Alcohol Review 1999; 18: 305-315

41 Schmidt-Semisch H. Überlegungen zu einem legalen Zugang zu Heroin für alle. Kriminologisches Journal 1990; 1 (90): 122-139

42 Strathdee SA, van Ameijden EJC, Mesquita F, Wodak A, Rana S, Vlahov D. Can HIV epidemics among injection drug users be prevented? AIDS 1998; 12: S71-S79

${ }^{43}$ Swiss Federal Office of Public Health. Swiss Methadone Report. Berne/Toronto: Swiss Federal Office of Public Health/Addiction Research Foundation, 1997; ISBN 0-88868-277-8

44 Uchtenhagen A, Dobler-Mikola A, Steffen T, Gutzwiller F, Blättler R, Pfeifer S. Prescription of Narcotics for Heroin Addicts? Main Results of the Swiss National Cohort Study, Basel: Karger Verlag, 1999

45 United Nations International Drug Control Programme (UNDCP). World Drug Report. Oxford: Oxford University Press, 1997
Prof. Dr. Jürgen Rehm

Institut für Suchtforschung

Konradstraße 32

8005 Zürich

Schweiz

E-mail: jtrehm@aol.com 